\title{
Circulating Angiogenic Factors, Tumor Cells, and Outcome after Resection of Metastatic Colorectal Cancer-What Does It Mean?
}

\author{
Alexander A. Parikh, MD ${ }^{1}$ and Lee M. Ellis, $\mathrm{MD}^{2}$ \\ ${ }^{1}$ Division of Surgical Oncology, Vanderbilt University Medical Center, Nashville, TN; ${ }^{2}$ Departments of Surgical Oncology \\ and Cancer Biology, The University of Texas M. D. Anderson Cancer Center, Houston, TX
}

The treatment of hepatic metastases from colorectal cancer (CRC) has undergone marked changes in the past decade. Improvements in surgical technique combined with more effective systemic therapies have led to a more aggressive approach for patients with CRC hepatic metastases. However, despite incremental advances with the addition of targeted agents to chemotherapy backbones, it is clear that our understanding of the biologic and molecular alterations that lead to metastases and resistance to therapy remains suboptimal.

Patients who undergo liver resection for hepatic metastases from $\mathrm{CRC}$ experience recurrence rates ranging 60-85\%. ${ }^{1}$ Because several therapeutic options now exist in these patients, it has become increasingly important to identify those patients at highest risk for recurrence, and it is equally important to better understand the biology of the tumor to better tailor treatment strategies.

In the current issue of Annals, Rahbari et al. reported on the expression and correlation of circulating angiogenic factors (CAFs) with disease recurrence in patients with metastatic CRC (mCRC) undergoing potentially curative hepatic resection. ${ }^{2}$ In their study of 107 patients, serum levels of eight CAFs and circulating tumor cells were measured. By multivariate analysis, low levels of placental growth factor (PlGF) and a Memorial Sloan-Kettering Cancer Center (MSKCC) clinical score of $>2$ were independent predictors of poor recurrence-free survival. Several other angiogenic factors, including serum vascular endothelial growth factor (VEGF), epidermal growth factor, interleukin 8 , and others did not predict recurrence in this study. In addition, data on circulating tumor cells were

(C) Society of Surgical Oncology 2011

Published Online: 28 May 2011

A. A. Parikh, MD

e-mail: alexander.parikh@vanderbilt.edu not available in $>40 \%$ of the patients, precluding its inclusion into their multivariate model.

Molecular markers have been proposed as predictors of response, recurrence, and survival. CAFs, for example, have been reported to correlate with clinicopathologic characteristics of various solid organ tumors, including stage of disease and prognosis - although these results have not been consistent. ${ }^{3,4}$ For example, several studies have suggested a relationship between angiogenic factors such as VEGF, PlGF, and overall outcome in patients with $\mathrm{CRC}^{4}{ }^{4}$ In addition, in a recent phase 2 trial of infusional 5-fluorouracil, irinotecan, and bevacizumab (FOLFIRI-B), elevated levels of interleukin- 8 were associated with a shorter progression-free survival. ${ }^{3}$ In this same study, several CAFs associated with angiogenesis and myeloid recruitment increased before radiographic evidence of progression including bFGF, HGF, SDF-1, MCP-3, and PlGF (which is in contrast to the current study). Although additional studies and validation are needed, these results suggest that measurement of circulating factors such as CAFs may predict and therefore lead to earlier therapeutic intervention, such as adjuvant therapy. However, it is important to note that high risk of recurrence does not correlate with benefit from adjuvant therapy. In future clinical trials, it will be important to stratify patients a priori according to risk of recurrence so that we may be able to prospectively evaluate the effect of therapy in preplanned subset analyses, which would certainly be more credible than any post hoc unplanned analyses.

The finding that low levels of PIGF correlated with poorer recurrence-free survival is puzzling and in contrast to other studies, which found that higher levels of PlGF were present before progression before decreasing somewhat at the time of progression. ${ }^{3}$ The authors note that PIGF levels were lower in healthy controls (versus patients with $\mathrm{mCRC}$ ) as well as in patients without recurrent 
metastases, which is not concordant with their finding that low levels of PIGF was actually associated with a poorer outcome. The comparisons were based on the 90th percentile, and when the multivariate analysis was performed with PlGF used as a continuous variable, the hazard ratio was actually 1.0 (95\% confidence interval 0.99-1.0), making this finding difficult to interpret. This emphasizes the importance of standardization and validation of both the assay and the analysis of the comparison groups (including statistical methods), particularly before utilizing them in the clinical setting. For example, in the United States, any assays that have an impact on patient care are required to be performed in Clinical Laboratory Improvements Amendments-certified laboratories.

Several other strategies have evolved in an attempt to predict both tumor recurrence rates and survival after hepatic resection of CRC metastases. Traditionally, these have focused on clinical and pathologic characteristics of the tumor, and several different clinical risk scores have been developed to predict recurrence and outcome. ${ }^{5}$ In 1999, Fong et al. from MSKCC published a clinical risk score encompassing five clinical criteria: nodal status of the primary tumor, disease-free interval from the primary tumor to the development of liver metastases in $<12$ months, number of tumors $>1 \mathrm{~cm}$ in size, preoperative carcinoembryonic antigen level $>200 \mathrm{ng} / \mathrm{ml}$, and size of tumor $>5 \mathrm{~cm}$ were chosen as part of the score. ${ }^{6}$ The total MSKCC score was noted to be highly predicative of longterm outcome and recurrence, and has been validated in several subsequent studies. ${ }^{5,7-9}$

The findings of the current study-that the MSKCC score and potential levels of PIGF independently predict disease outcome-suggest that both clinical and molecular parameters may be useful and complementary in predicting disease recurrence. Nevertheless, several other questions remain. For example, although the authors attempted to control for confounding factors in their model, only the MSKCC clinical score and number of metastases $>1$ were included in their multivariate analysis. Other clinical parameters were used in their univariate analysis only, and several of these are already a part of the MSKCC score. In addition, although the authors note that excluding patients who received neoadjuvant bevacizumab did not alter their results, details of other therapeutic regimens are not known. Adjuvant therapy, for example, was administered at the discretion of the medical oncologist and was provided in $>50 \%$ of patients. Because the details of these regimens-including the type of agents provided, number of cycles of therapy, and timing of therapy-were not known, this was not included in their model. The use of adjuvant therapy after resection of primary CRCs is the standard of care for most patients, and many other studies (although mostly nonrandomized) have suggested a possible role for adjuvant therapy after liver resection. ${ }^{10}$ Furthermore, it is unclear whether CAF levels themselves are affected by cytotoxic chemotherapy regimens, which were administered in in the neoadjuvant setting in $33 \%$ of patients. ${ }^{3}$ An in vitro study by Fan et al., for example, showed that acute administration of oxaliplatin can induce several members of the VEGF family of ligands, including VEGF and PlGF. ${ }^{11}$ Thus, the use and type of neoadjuvant therapy (or even adjuvant therapy) may be a statistically significant confounding variable and should be included in future analyses.

Single-institution retrospective studies are important because they are hypothesis-generating studies. However, it is essential to recognize the limits of such studies and until validation is obtained in larger, prospective, well-controlled studies conducted at multiple sites. For all studies that evaluate prognostic markers, one must consider how this will impact day-to-day practice- - that is, when a patient is sitting across from the surgeon or oncologist in the clinic or office, how will the clinician utilize this information to alter patient care and thus outcome? Measurement of CAFs, although not the standard of care at this time, can be easily done if found to be of clinical value. However, as stated above, one must not apply this knowledge to clinical practice until confirmed in prospective multicenter trials.

In summary, the use of molecular markers such as CAFs in predicting recurrence rates and survival in patients with metastatic disease is certainly intriguing, and evidence to date suggests it may play a complementary role to other clinical parameters. However, additional well-designed studies are needed to help clarify their clinical significance and relevance. In the design of these studies, every attempt must be made to control for the myriad of confounding factors that affect survival in these patients, particularly the details of the treatment regimens given.

\section{REFERENCES}

1. de Jong MC, Pulitano C, Ribero D, et al. Rates and patterns of recurrence following curative intent surgery for colorectal liver metastasis: an international multi-institutional analysis of 1669 patients. Ann Surg. 2009;250:440-8.

2. Rahbari NN, Reissfelder C, Mühlbayer M, et al. Correlation of circulating angiogenic factors with circulating tumor cells and disease recurrence in patients undergoing curative resection for colorectal liver metastases. Ann Surg Oncol. doi:10.1245/s10434011-1761-9.

3. Kopetz S, Hoff PM, Morris JS, et al. Phase II trial of infusional fluorouracil, irinotecan, and bevacizumab for metastatic colorectal cancer: efficacy and circulating angiogenic biomarkers associated with therapeutic resistance. J Clin Oncol. 2010;28: 453-9.

4. Kwon KA, Kim SH, Oh SY, et al. Clinical significance of preoperative serum vascular endothelial growth factor, interleukin-6, and C-reactive protein level in colorectal cancer. BMC Cancer. 2010;10:203 
5. Feroci F, Fong Y. Use of clinical score to stage and predict outcome of hepatic resection of metastatic colorectal cancer. J Surg Oncol. 2010;102:914-21.

6. Fong Y, Fortner J, Sun RL, Brennan MF, Blumgart LH. Clinical score for predicting recurrence after hepatic resection for metastatic colorectal cancer: analysis of 1001 consecutive cases. Ann Surg. 1999;230:309-18.

7. Arru M, Aldrighetti L, Castoldi R, Di Palo S, et al. Analysis of prognostic factors influencing long-term survival after hepatic resection for metastatic colorectal cancer. World J Surg. 2008;32: 93-103.

8. Mala T, Bohler G, Mathisen O, Bergan A, Soreide O. Hepatic resection for colorectal metastases: can preoperative scoring predict patient outcome? World J Surg. 2002;26:1348-53.
9. Mann CD, Metcalfe MS, Leopardi LN, Maddern GJ. The clinical risk score: emerging as a reliable preoperative prognostic index in hepatectomy for colorectal metastases. Arch Surg. 2004;139: $1168-72$.

10. Power DG, Kemeny NE. Role of adjuvant therapy after resection of colorectal cancer liver metastases. J Clin Oncol. 2010;28: 2300-9.

11. Fan F, Gray MJ, Dallas NA, et al. Effect of chemotherapeutic stress on induction of vascular endothelial growth factor family members and receptors in human colorectal cancer cells. Mol Cancer Ther. 2008;7:3064-70. 